Case Report

\title{
Moyamoya Disease Presenting as Ischemic Stroke Following Heamorrhagic Strokein a 46-year-old Man: A Case Report
}

\author{
*Majumder $\mathrm{SN}^{1}$, Sheikh $\mathrm{AK}^{2}$, Jahangir $\mathrm{M}^{3}$, Chowdhury $\mathrm{R}^{4}$
}

\begin{abstract}
Moyamoya is a rare cerebrovascular disease of unknown etiology. It can affect both children and adults. Ischemic symptoms are common in younger age while adults presents with intracranial hemorrhage. Cerebral ischemia after hemorrhage within a narrow time frame or simultaneous presentation with both hemorrhage and ischemia in the same clinical setting is a rare encounter. Diagnosis is confirmed by doing cerebral angiogram. Here, we report a case of 46-year-old man who presented with hemiparesis and imaging of brain showed ishaemic stroke initially and subsequently he also developed haemorrhagic stroke. Later, magnetic resonance imaging and digital substraction angiogram of brain confirmed Moyamoya disease. He was managed conservatively with significant improvement of his hemiparesis.
\end{abstract}

Keywords: Moyamoya disease, cerebral infarction, hemorrhagic stroke

\section{INTRODUCTION}

Moyamoya disease is a chronic progressive and occlusive disorder of cerebral arteries which is characterized by angiogenesis where the brain attempts to compensate for ischemia by producing a local network of tiny blood vessels, which appear cloud-like on angiograms. ${ }^{1}$

Moyamoya disease (MD) was first reported in Japan in 1957. ${ }^{2} \mathrm{MD}$ is frequently reported in East Asia where its

1. *Dr. Sabbiha Nadia Majumder, Specialist Registrar, Neurology, Square Hospitals Limited, Phone no: 01682076378 E-mail:snadiamajumder@gmail.com

2. Dr. Abdul Kader Sheikh, Consultant, Neurology, Square Hospitals Limited

3. Dr. Mehjabeen Jahangir, Residential Medical Officer, ICU, Square Hospitals Limited

4. Dr. Romana Chowdhury, Phase-B Resident, Department of transfusion Medicine, BSMMU, Dhaka

${ }^{*}$ For correspondence incidence is more than 10 times that in western countries. ${ }^{3}$

It has been found in all races with varying age distributions and clinical manifestations. ${ }^{4}$ Both children (aged 0-10 years) and adults (aged 30-40 years) are affected. ${ }^{4}$

Clinically, symptoms of brain ischemia are usually found in MD children, while transient or permanent brain infarction and intracranial hemorrhage are noted in MD adults. $^{5}$ Presence of cerebral infarction along with parenchymal hemorrhage in adult with bleeding-type Moyamoya disease is reported rarely in literature.

We described the case of a 46-year-old gentleman having been diagnosed with moyamoya disease presented with brain ischemia after receiving initial diagnosis of intracerebralhemorrhage.

\section{CASE REPORT}

This 46-year-old hypertensive and newly diagnosed diabetic gentleman presented to the emergency department of Square Hospital Limited with the complaints of right sided weakness of both upper and lower limbs for 9 days Weakness initially appeared over right upper limb which evolved into lower limb gradually. There was no distant history of head injury, altered sensorium or convulsion. He was non smoker and non alcoholic. There was no significant family history of such illness. His only medication of note included calcium channel blocker. General examination was notable for a high blood pressure that was $160 / 80 \mathrm{mmhg}$. His BMI was found to be $37 \mathrm{~kg} / \mathrm{m} 2$. Positive findings of neurological examination were confined to the right side revealing diminished tone, muscle power $3 / 5$ over both upper and lower limbs, exaggerated tendon reflexes with extensor plantar response. All modalities of sensation were intact and he had no signs of cerebellar involvement. Immediate CT scan of head depicted left sided evolving hematoma at periventricular and capsuloganglionic region without midline shift. (Figure-1) 


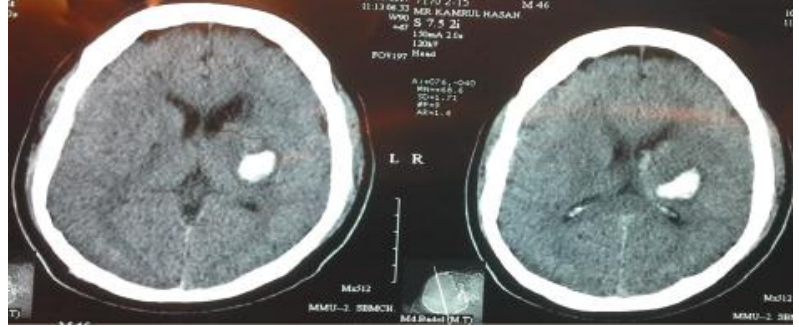

Figure-1: Haemorrhage in left sided capsuloganglionic region.

Preliminary blood workup showed no gross abnormality. Fundoscopic examination was insignificant. He was managed conservatively.

In the course of inpatient stay, the patient complained of visual disturbance. Subsequent visual field examination illustrated right sided homonymous hemianopia.

This newly appeared symptom prompted us to do follow up CT scan of Head which showed parieto-occipital-temporal lobesinfarct (new lesion) along with evolving hematoma on left side. (Figure-2) Moreover there was compression over the left ventricle and $4.2 \mathrm{~mm}$ midline shift to the right.

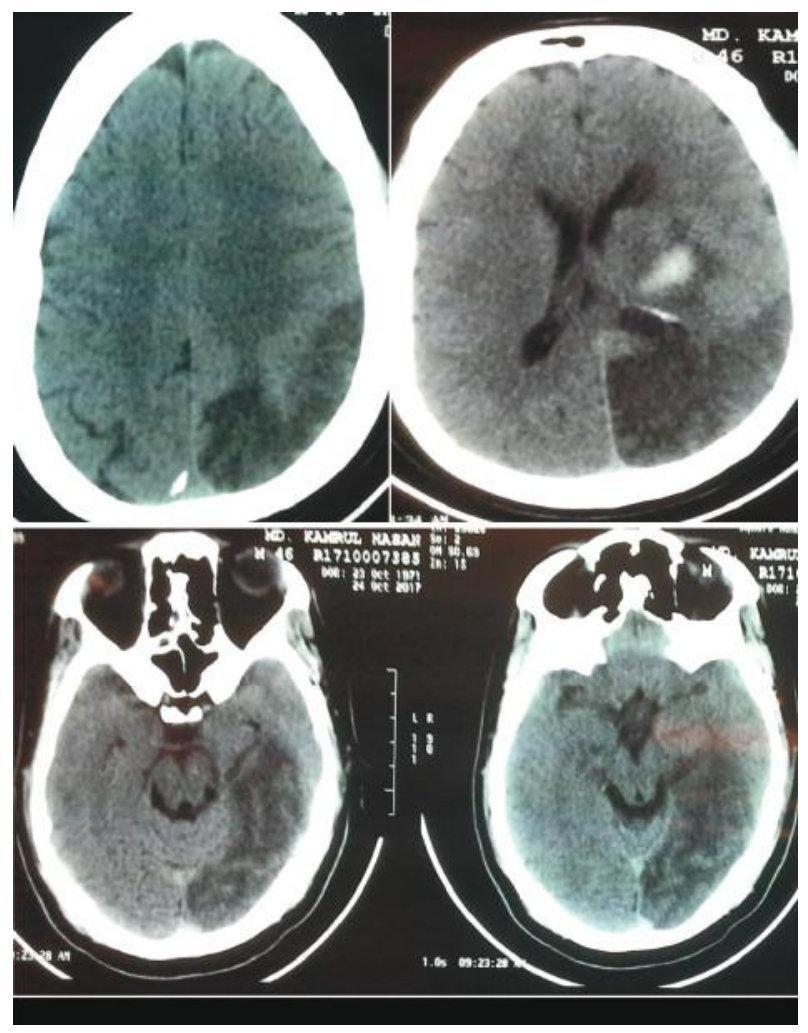

Figure-2: CT scan of head showing haemorrhage in left capsuloganglionic region and large infarction in left parieto-occipito- temporal region.
Subsequent MRI of brain revealed left sided large acute infarction as well. (figure-3)

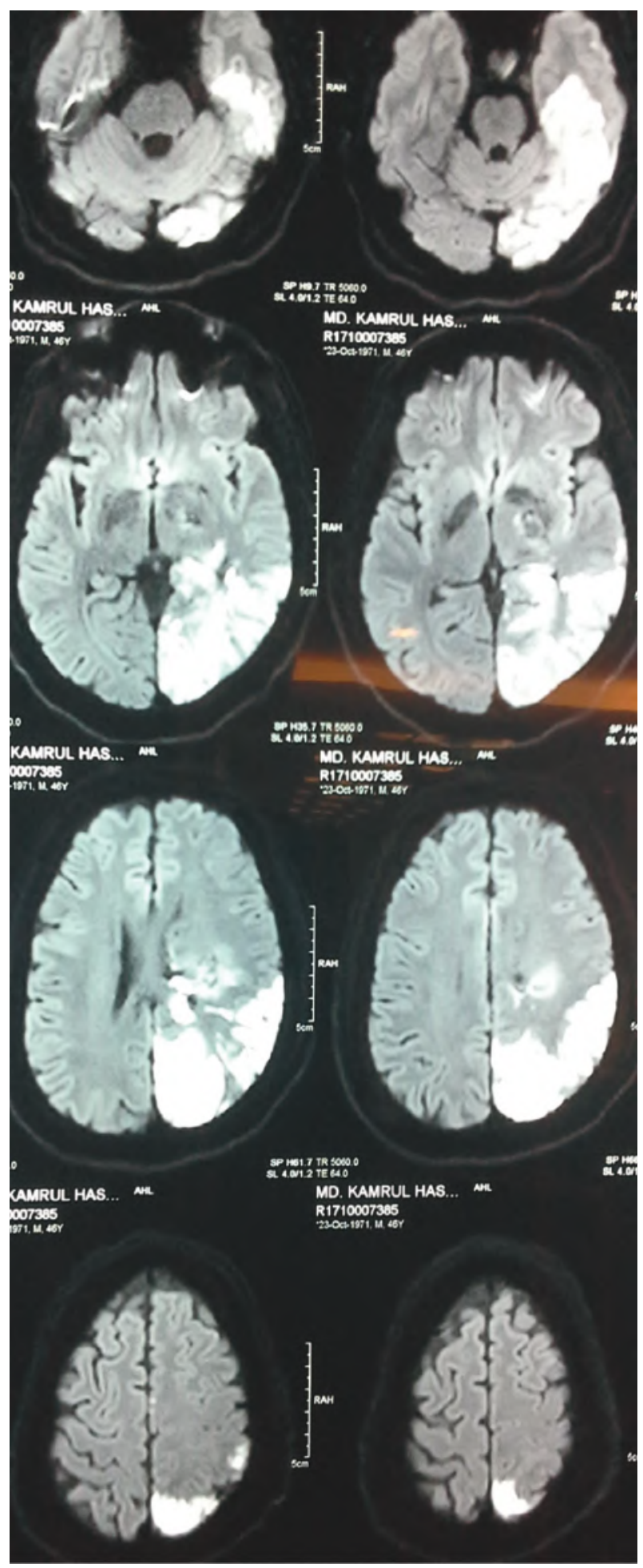

Figure-3: MRI of brain showing acute infarction in left parieto-occipito-temporal region. 
To exclude any vascular abnormality, we planned to carry out MR angiography. MRA report strikingly demonstrated supraclenoid part of the left ICAs having severe stenosis with numerous enlarged basal collateral vessels giving puff of smoke appearance while MCAs are also poorly visualized which is suggestive of Moya Moya disease with hypoplastic both posterior communicating artery and right vertebral artery (Figure-4).
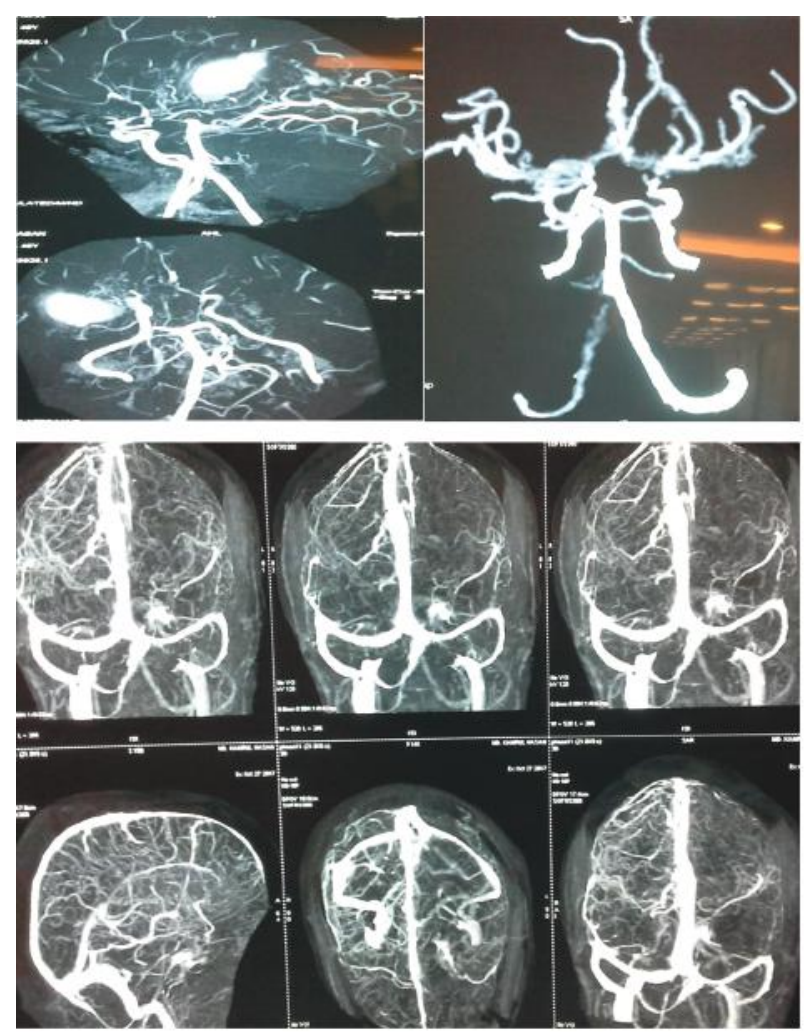

Figure-4: MRA of brain showing supraclenoid part of the left ICAs having severe stenosis with numerous enlarged basal collateral vessels giving puff of smoke appearance and MCAs are also poorly visualized.

Meanwhile within few days his right sided muscle power remarkably improved to $4 / 5$ and visual clarity was restored to some extent. After that, CT angiogram with contrast was done which was evident of severe stenosis of supraclenoid portion of both internal carotid artery and MCA with incomplete circle of willis formation associated with abnormal vascular network in the vicinity of occlusion/stenosis- favouring Moyamoya disease. Both posterior communicating arteries and right vertebral artery were not visualized.

Eventually DSA was performed showing total occlusion of distal RICA, proximal MCA and near total occlusion of
ACA after RICA injection. Furthermore LICA injection revealed that there was total occlusion of distal LICA and proximal ACA and near total occlusion of proximal MCA. P1 of right PCA was dilated. From PCA, cortico-cortical anastomosis was formed to the area of right MCA. Final comment was suggestive of Moyamoya disease ( Suzuki Grade 4).Neurosurgical opinion was taken about EC-IC bypass. The patient declined to do any intervention. $\mathrm{He}$ was discharged with conservative treatment and follow up visit showed significant improvement of his weakness nevertheless residual visual problem persisted. No noticeable deterioration was observed.

\section{DISCUSSION}

Moyamoya disease is a poorly understood occlusive disease involving large intracranial arteries, specially the distal internal carotid artery and the stem of the MCA and ACA. The lenticulostriate arteries develop a rich collateral circulation around the occlusive lesion which gives the impression of a 'puff of smoke' on conventional x-ray angiography. Other collaterals include transdural anastomoses between the cortical surface branches of the meningeal and scalp arteries. ${ }^{6}$

Moyamoya is a Japanese word for 'haze' it has been used to refer to an extensive basal cerebral rete mirabile- a network of small anastomotic vessel at the base of the brain around and distal to the circle of Willis seen in carotid angiogram associated with stenosis of intracranial arteries. ${ }^{7}$ The steno-occlusive areas are usually bilateral, but unilateral involvement does not exclude the diagnosis. ${ }^{8}$ Moyamoya is a rare disease with reported incidence of 0.086 per 100,000 population. ${ }^{9}$

Moyamoya was originally considered to affect predominantly persons of Asian heritage specially in Japan but has now been observed throughout the world. The incidence peaks lie within two age groups: children who are 5 years old and adults in their mid 40 's. ${ }^{10}$ Our patient was a 46-year old man which coincided with the adult peak age group.

The disease is believed to be genetic. Certain associations like thyroid abnormalities, hemoglobinopathies, down syndrome are found with this clinical entity. ${ }^{8}$ Moyamoyadisease is categorized as either ischemic and hemorrhagic type or true mixed type. The clinical presentations and course differ with various age group. Adults experience intracranial hemorrhage including intracerebral hemorrhage (ICH), intraventricular 
hemorrhage (IVH), and subarachnoid hemorrhage (SAH) more commonly; cerebral ischemic events like transient ischemic attack (TIAs), cerebral infarction are more common in children. Patient may remain asymptomatic as well. $^{8}$

The risk of ischemic complications in patients with bleeding-type Moyamoya disease is poorly understood with a few cases reported, mainly in adults. ${ }^{11}$ Our case was presented with large ischemic stroke on top of the acute phase of bleeding type moyamoya disease.

Breakdown of dilatedlenticulostriate arteries may produce parenchymal hemorrhage and progressive occlusion of large surface arteries can occur, producing large-artery distribution strokes. ${ }^{6}$ It is assumed that similar incidence happened in our case. Bleeding in the periventricular and capsuloganglionic region could be due to rupture of dilated lenticulostriate arteries. In addition, hypertension may be a contributing factor. The mechanism of ischemia may be varied in each individual patient. ${ }^{12}$ The effects of ICH on cerebral hemodynamics are increased ICP. Raised ICP may cause ischemia. ${ }^{13}$ Even administration of hyperosmolar drug for reduction of ICP sometimes results in dehydration. ${ }^{14}$

Hypotension, dehydration and even vasospasm in patients with ICH may cause irreversible cerebral ischemia in Moyamoya disease as cerebral hemodynamic conditions may be critical after ICH. The risk factors for progression to cerebral ischemia are complex and cerebral hemodynamics are difficult to maintain in the acute phase of bleeding type of Moyamoya disease. ${ }^{12}$

No clinical features of raised ICP, dehydration were observed in our patient. Patient was hemodynamically stable throughout the clinical course following intracerabral bleed. There was no midline shift or features of cerebral edema in the initial CT scan as well. So it is difficult to draw conclusion regarding exact mechanism behind ischemia.Total occlusion of distal LICA and proximal ACA and near total occlusion of proximal MCA seen on DSA might be responsible for large artery territory ischemic stroke.

The Suzuki staging system for moyamoya disease refers to findings on conventional angiography. According to the Suzuki classification system, MD was graded as I to VI . Moreover, grade I and II was defined as early stage, grade III and IV as intermediate stage and V and VI as late stage. The incidence of grade IV and V MD in hemorrhagic MD was higher than in ischemic MD. ${ }^{15}$
DSA findings of our case are consistent with the stage 4 disease.

Conventional angiography is the gold standard for both the diagnosis and surgical planning for patients with suspected moyamoyadisease. ${ }^{6}$ Medical treatment is far from satisfactory. Revasularization procedures are gaining importance as a primary treatment for Moyamoya. ${ }^{16}$ Employment of surgical intervention depends on patient's condition.

\section{CONCLUSIONS}

The simultaneous findings of intracerebral bleeding and ischemia in the CT scan of Head should lead us to think about moyamoya disease. It is a less common clinical presentation but not rare.

\section{REFERENCES}

1. Jeremy Hertza, Ashlee Loughan, Robert Perna, Andrew S. Davis, Kelly Segraves\& Nina L. Tiberi. Moyamoya Disease: A Review of the Literature, Applied Neuropsychology: Adult,2014; 21(1): 21-27

2. Takeuchi K, Shimizu K. Hypoplasia of the bilateral internal carotid arteries. Brain Nerve. 1957;7:37-43.

3. Hayashi K, Horie N, Suyama K, Nagata I. An epidemiological survey of moyamoya disease, unilateral moyamoya disease and quasi-moyamoya disease in Japan. ClinNeurolNeurosurg. 2013; 115:930-33.

4. Janda PH, Bellew JG, Veerappan V.Moyamoya disease: case report and literature review, J Am Osteopath Assoc. 2009;109(10):547-53

5. Hayashi K, Horie N, Izumo T, Nagata I. A nationwide survey on unilateral moyamoya disease in Japan. ClinNeurolNeurosurg. 2014;124:1-5

6. Kasper, D. L., Fauci, A. S., Hauser, S. L., Longo, D. L. 1., Jameson, J. L., \&Loscalzo, J. Harrison's principles of internal medicine.19th ed. McGraw Hill Education:New York;2015

7. Ropper, A. H., ADAMS, R. D., VICTOR, M., BROWN, R. H., \& VICTOR, M. Adams and Victor's principles of neurology. $12^{\text {th }}$ ed. McGraw-Hill Medical Pub.Division. New York;2005

8. Roy Sucholeiki, MD; Amy Kao,.Moyamoya Disease. Medscape ,2017.Sep.06 
9. Uchino K, Johnston SC, Becker KJ, et al. : Moyamoya disease in Washington State and California. Neurology. 2005;65

10. Han DH, Kwon OK, Byun BJ, et al. : A co-operative study: clinical characteristics of 334 Korean patients with moyamoya disease treated at neurosurgical institutes (1976-1994). The Korean Society for Cerebrovascular Disease. ActaNeurochir (Wien). 2000;142(11):1263-73.

11. Kim DS, Jang DK, Huh PW, Yoo DS, Han YM, Huh CW. Ischemic stroke after acute intracranial haemorrhage in patients with Moyamoya disease: Six new cases and a short literature review. ActaNeurochir (Wien) 2011:1253-61 Jun;153(6):1253-63

12. Iwama $T$, Kotani $Y$, Yamakawa $H$, Nagata I, Hashimoto N, Sakai N. Cerebral ischemic complication following intracranial bleeding in patients with Moyamoya disease-three case reports. Neurol Med Chir (Tokyo) 2001;41:450-3

13. Iwama T, Hashimoto N, Tsukahara T, Murai B. Perioperative complications in adult Moyamoya disease. ActaNeurochir (Wien) 1995;132:26-31.

14. Iwama T, Hashimoto N, Yonekawa Y. The relevance of hemodynamic factors to perioperative ischemic complications in childhood Moyamoya disease. Neurosurgery. 1996;38:1120-6.

15. Suzuki J, Takaku A. Cerebrovascular "moyamoya" disease. Disease showing abnormal net-like vessels in base of brain. Arch Neurol. 1969;20:288-299.

16. Fung LW, Thompson D, Ganesan V: Revascularization surgery for pediatric moyamoya: a review of the literature. Childs Nerv Syst. 2005; 21(5):358-64. 